\title{
Genitourinary syndrome of menopause - is the problem solved? State of the art 2018
}

\author{
Jacek Szymański, Kornelia Zaręba, Grzegorz Jakiel, Aneta Słabuszewska-Jóźwiak \\ $1^{\text {st }}$ Department of Obstetrics and Gynecology, Centre of Postgraduate Medical Education, Warsaw, Poland
}

\begin{abstract}
Vulvovaginal atrophy accompanied by lower urinary tract dysfunction related to low levels of estrogen and androgens is labeled as genitourinary syndrome of menopause (GSM). Although this condition affects most postmenopausal women worldwide, it seems to be underdiagnosed and undertreated. Women should be properly advised to choose an adequate treatment modality to improve their quality of life, sexual relationships and social activity. The aim of this article to is increase knowledge of GSM. The current treatment options, both hormonal and non-hormonal, are reviewed. Topical estrogen therapy still remains the gold standard, but the demand for individually tailored therapy is growing. New treatment modalities are continuously included in clinical practice. They should consider the whole personality of a woman as well as cultural and social factors. Further studies on GSM and on the effectiveness of various treatment options are necessary to achieve this purpose.
\end{abstract}

Key words: menopause, vulvovaginal atrophy, androgens, estrogens, SERMs, laser, RF devices.

\section{Introduction}

Vulvovaginal atrophy (VVA) accompanied by lower urinary tract dysfunction (LUTS) related to low levels of estrogen and androgens is labeled as genitourinary syndrome of menopause (GSM). The signs and symptoms of VVA - vaginal dryness, irritation, burning and dyspareunia -are the most bothersome for postmenopausal women. The GENISSE study revealed presence of GSM in $70 \%$ of participants. The most prevalent signs were vaginal dryness (93.3\%), reduced lubrication (90\%) and dyspareunia (81.6\%), markedly decreasing quality of life [1]. However, as it was shown in the AGATA study, there is great confusion about the therapy of VVA. The treatment is heterogeneous and inadequate. The discontinuation rate is almost $40 \%$. Women spontaneously withdraw from treatment due to the lack of efficiency $(46.6 \%)$, messiness $(24.3 \%)$, difficulty in application (7.8\%) and vaginal discharge (1.9\%). The absence of clear therapeutic standards based on wellcontrolled clinical studies leads to heterogeneity of the treatment schedules.

Inadequate prescribed products in terms of type, time schedule and length of administration make the therapy insufficient and decrease a patient's compliance. In fact, VVA remains practically untreated [2]. These problems seem to affect women all over the world. The European REVIVE study (REVIVE-EU) showed that $47 \%$ of participants were not aware of the VVA condition, $8.1 \%$ were not aware of the cause of VVA and only $39.9 \%$ directly attributed VVA to the menopause. The European women mostly suffered from vaginal dryness (70\%), vaginal irritation (32.7\%), dyspareunia (29\%) and vaginal tenderness (14.3\%). A great impact of VVA symptoms on sexual satisfaction was reported by $72 \%$ of participants. More than $60 \%$ of women in the survey discussed these problems with their HCP (health care professional), but about 50\% had to initiate this conversation themselves. Satisfaction with treatment was reported by approximately $45 \%$ of women and the OTC personal lubricants and moisturizers were the most common agents. The therapy was discontinued by $39.2 \%$ of participants, mainly because the symptoms were not bothersome enough to continue treatment (18.4\%), the medication was not sufficiently effective (18.3\%), or due to relief from VVA symptoms. The main worries related to vaginal hormonal agents were concerns about adverse effects (AE) (55.7\%) and a fear of possible carcinogenesis [3]. The pan-Asian REVIVE survey gave evidence that GSM is underdiagnosed and undertreated in Asia. Only 25\% of women discussed their VVA symptoms with a healthcare professional and only $24 \%$ were HCP-initiated. Moreover, only $21 \%$ of the participants were clinically diagnosed with GSM by HCP. The most common rea- 
son for not discussing GSM symptoms with a HCP was the belief that these symptoms were a natural part of aging (49\%) and would diminish over time without treatment. Among women who have ever been treated $52 \%$ abandoned therapy because they felt improved or cured. The users of GSM treatment reported a high degree of concern mostly related to hormonal therapy and its safety in long-term use (58\%) [4]. The VIVALATAM study revealed the lack of knowledge of postmenopausal vaginal atrophy in women of Latin America. Across Latin America there are huge differences between countries in health care access and standards. Some country-specific differences between surveyed populations of women were confirmed in this study. For instance, fewer responders in the Brazilian cohort were concerned about the health of their vagina $31 \%$ [64\% overall]) and their sexual health (27\% [51\% overall]). More women from Columbia associated VVA with a symptom of the menopause (54\% [ $45 \%$ overall]). For women from Chile urinary incontinence constituted the major problem (75\% [60\% overall]). Nevertheless, overall $71 \%$ of the participants did not know vaginal atrophy to be a chronic condition and only 6\% attributed vaginal dryness, itching, burning or dyspareunia directly to VVA. Overall $64 \%$ of the surveyed population believed that vaginal discomfort could limit the comfort of their everyday activities, including sex, and 56\% considered that the condition could also decrease the comfort of sexual satisfaction of their partners. Despite the availability of many treatment modalities, 34\% of women with severe vaginal symptoms do not believe in the possibility of effective therapy. However, 52\% of women were aware of the existence of some form of local hormonal treatment [5]. Similar studies from other countries and world regions show that despite cultural differences, the GSM is underdiagnosed and undertreated. Women need more information, more education and wider access to treatment modalities to improve their sexual functions and postmenopausal quality of life [6].

\section{Androgens}

During menopause, estrogen synthesis in the ovaries ceases rapidly, but this process is not directly accompanied by a parallel decrease of the androgen blood levels. The androgens decrease progressively throughout adult life, but a lower sex hormone-binding globulin (SHBG) concentration in the menopause period allows the free testosterone level to be kept stable [7]. Androgens act by activation of the intracellular androgen receptors (AR), which are localized in the vaginal tissues, vestibule, clitoris, labia, bladder, urethra and pelvic muscles [8]. AR stimulation could involve the genomic pathway, through androgen response elements (AREs) on the DNA, resulting in mRNA expression of specific androgen-responsive genes leading to changes in cellular metabolism or non-genomic signaling related to the cytoplasmic and membrane-associated receptor activation [9]. After menopause dehydroepiandrosterone (DHEA) becomes the main source of the androgens and estrogens synthesized in peripheral tissues. The blood concentration of DHEA declines continuously from the third decade of life and leads to a decrease in the peripheral synthesis of sex steroids after menopause. This, in consequence, causes the constellation of perimenopausal signs and symptoms, such as hot flushes, sexual dysfunction, VVA, bone and muscle loss, cognition disorders and many others. An intracrinological enzymatic system unique to humans maintains under control intracellular transformation of DHEA into active sex steroids. The UGT enzymes (the uridine diphosphate [UDP]-glucuronosyltransferase family), involved in the glucuronidation pathway, maintain the sex steroids serum level within the normal postmenopausal range. Thus, activation of the sex hormone specific tissue receptors is strongly limited $[10,11]$. Intravaginal 0.5\% (6.5 mg) DHEA has no significant effect on serum estrogens and androgens levels. The serum $\mathrm{E}_{2}$ concentration 12 weeks after intravaginal administration of prasterone was about 19\% below the postmenopausal value [12]. Contrarily, long-term local treatment with low doses of E2 could increase the serum estradiol level with possibly systemic estrogenic effects [13]. A recent review of 12 randomized controlled trials of intravaginal prasterone versus placebo revealed safety and high effectiveness of DHEA in VVA therapy. At the 52week follow-up of daily treatment of $0.5 \%$ prasterone (6.5 mg) a significant improvement in desire, arousal, lubrication, orgasm, sexual satisfaction and pain scores was observed [14]. Cytological analysis showed a significant decrease in parabasal vaginal epithelial cells with simultaneous increase in superficial layer and vaginal secretion [15]. In any case, where an impact of DHEA on endometrium was analyzed, a proliferative effect was found [16]. A comparison of intravaginal $6.5 \mathrm{mg}$ prasterone with $0.3 \mathrm{mg}$ conjugated estrogens and $10 \mu \mathrm{g}$ estradiol showed at least equal efficacy of DHEA as vaginal estrogens [17]. The most commonly reported AE were vaginal discharge $(5.71 \%)$ and abnormal cervical smear (in $2.1 \%$ of women: low-grade squamous intraepithelial lesions [L-SIL] and atypical cells of undetermined significance [ASC-US]) [18].

\section{Low-dose vaginal estrogens}

Low-dose vaginal estrogens constitutes the gold standard of VVA therapy. In the statement of the North American Menopause Society (NAMS), this treatment is preferred over systemic estrogen therapy due to its safety and effectiveness [19]. The wide range of FDAapproved products help patients to choose the most 
suitable, accepted and convenient form. The optimal treatment should involve the lowest dose of vaginal estrogen which makes the therapy effective. Estrogen vaginal creams, inserts and rings are available in the USA. The vaginal cream, applied 0.5-1.0 g daily for 2 weeks, followed by 0.5-1.0 g 1-3 times weekly, contains estradiol-17 $\beta$ or conjugated estrogens (conjugated estrogens are not available on the European market). It provides additional vaginal moisturizing but the lack of a dosing unit increases the risk of exceeding the proper dose. Vaginal tablets (10 mg estradiol) support more controlled dosing of $E_{2}$. Vaginal softgel capsules with estradiol-17 $\beta$ (4, 10 and $25 \mathrm{mg}$ doses) are a novel promising option for VVA treatment. Two randomized, single-dose, two-way cross-over trials comparing the pharmacokinetics of a solubilized estradiol softgel capsule with a vaginal estradiol tablet in postmenopausal women at $10 \mathrm{mg}$ and $25 \mathrm{mg}$ doses established the safety, patient's satisfaction and good toleration of the softgels. Serum levels of estradiol, estrone and estrone sulfate were significantly lower than those found with the estradiol $10 \mathrm{mg}$ vaginal tablet [13]. A recent randomized, multicenter, double-blind study showed the effectiveness of a very low-dose estradiol vaginal cream $(0.003 \%)$ applied twice a week in the treatment of vaginal dryness [20]. Mueck et al. in a clinical review revealed the efficacy of ultra-low doses of estriol $(0.03 \mathrm{mg} / \mathrm{l})$ in combination with Lactobacillus acidophilus in restoring vaginal mucosa and microflora in ageing menopausal women [21]. Santen compared various doses of estrogens in local vaginal therapy and their concentration in the serum. He noted that the estrogen blood level did not exceed the postmenopausal value $(<20 \mathrm{pg} / \mathrm{ml})$ during long-term administration of low-dose estrogens (e.g. $10 \mathrm{mg}$ vaginal tablets). The intermediate and high dose-estrogen regimens $(25 \mathrm{mg}$ and higher estradiol agents) maintained the plasma estrogen concentration within the premenopausal range [22]. There are two vaginal rings available releasing different estrogen doses, both applied for 90 days. The lower-dose ring contains $2 \mathrm{mg}$ of estradiol-17 $\beta$ and is dedicated for the treatment of VVA only. The higher-dose ring with $12.4 \mathrm{mg}$ of estradiol acetate provides a systemic hormone level adequate to the GSM and vasomotor symptoms therapy [19]. According to the NAMS and National Institute for Clinical Excellence (NICE), low-dose vaginal estrogens improve the vaginal maturation index, increase the number of vaginal lactobacilli and decrease vaginal $\mathrm{pH}$ [23]. The treatment should be continued as long as needed to relieve symptoms (NICE 2015). Moreover, the NAMS and NICE do not recommend the protection of endometrium with progesterone while treated with low-dose vaginal estrogens. However, monitoring of the endometrium is recommended in women with high risk of endometrial cancer and when higher doses of estrogen than usual are used in the therapy $[19,24]$ (NICE
2015). Currently animal studies are ongoing to evaluate the impact of estetrol $\left(E_{4}\right)$ (a weak estrogen produced exclusively by the human fetal liver during pregnancy) on vaginal tissue. Estetrol does not increase the level of hepatic-derived coagulation factors and thus should not increase the risk of thrombosis. The biological effect of $E_{4}$ is mediated by nuclear ER- $\alpha$ activation and it was found to increase vaginal epithelial proliferation and lubrication in ovariectomized mice [25].

\section{Selective estrogen receptor modulators}

Estrogen receptor agonist antagonists (ERAAs), previously called selective estrogen receptor modulators (SERMs), are nonsteroidal agents that selectively stimulate or inhibit estrogen receptors in different tissues. Ospemifene is the only ERAA approved by the FDA for the treatment of GSM. The effectiveness of ospemifene $60 \mathrm{mg}$ taken orally in the therapy of GSM was confirmed in 3 double-blind clinical trials. A significant improvement in dyspareunia and vaginal dryness was noted on the Likert scale after 12-week follow-up. Cytological examination revealed an improvement in the vaginal maturation index and a decrease in vaginal $\mathrm{pH}$ at weeks 12 and 52 [26-28]. Post hoc analysis of safety data pooled from six phase 2 and 3 RCTs revealed $A E$, mild and moderate in severity. The most commonly reported $\mathrm{AE}$ were hot flush ( $8.5 \%$ vs. $3.3 \%$ for placebo), urinary tract infection (6.5\% vs. $4.8 \%$ ), headache (5.4 vs. $5.9 \%$ ), nasopharyngitis (5.4\% vs. $3.1 \%$ ), muscle spasm (4.4\% vs. $1.4 \%$ ), vaginal discharge ( $4.4 \%$ vs. $0.4 \%)$, and vulvovaginal candidiasis ( $4.3 \%$ vs. $0.5 \%$ ). Discontinuation due to AE occurred in $7.6 \%$ of women treated with ospemifene versus $3.8 \%$ with placebo, and hot flushes were the most common reason. No detrimental impact on the breast, bone, and cardiovascular system was observed in women treated with ospemifene $60 \mathrm{mg}$ compared to placebo [29]. Moreover, the short- and long-term studies did not reveal any case of endometrial carcinoma and $<1 \%$ of patients experienced endometrial hyperplasia [30, 31]. Ospemifene is an optional treatment of dyspareunia in women who have not achieved benefits from personal lubricants and are not willing to use low-dose vaginal estrogens. Although ospemifene received marketing authorization in the UK, there is no clear NICE recommendation for administration of this medication in VVA treatment [32]. A positive effect on the genitourinary system was established for bazedoxifene $20 \mathrm{mg}$ in combination with conjugated estrogens $0.45 \mathrm{mg}$ approved by the FDA for the therapy of vasomotor symptoms and in prevention of postmenopausal osteoporosis [33]. The efficacy of these agents in the treatment of VVA was confirmed in the SMART-3 trial. A significant improvement in the vaginal maturation index, vaginal dryness, and decreased $\mathrm{pH}$ as compared to placebo were observed [34]. 


\section{Lubricants and moisturizers}

Women with vaginal dryness can benefit from using personal lubricants and moisturizers, especially for relief of pain during intercourse. A vast range of lubricants and moisturizers are available as OTC products. Lubricants are applied into the vagina before sexual intercourse, as they act rapidly, giving short-term relief from vaginal dryness. It seems that water-based lubricants are the most beneficial and acceptable by women with vaginal dryness. In contrast, the aim of vaginal moisturizers is to rehydrate mucosal tissue, so they should be applied regularly. Moisturizers adhere to the surface of the vaginal epithelial tissue, mimic natural secretion and maintain vaginal moisture due to epithelial hydration and acidifying the vaginal environment. In order to keep water in the vaginal lining the moisturizers contain either plant-based or synthetic polymers. Both lubricants and moisturizers also contain a wide range of additional ingredients such as humectants, emollients and preservatives to provide appropriate viscosity, $\mathrm{pH}$ buffering, alter water activity and prevent bacterial contamination [35]. All these excipients have an impact on osmolarity values and $\mathrm{pH}$. The World Health Organization (WHO) recommends the use of lubricants and moisturizers for women in the menopause and postmenopause [36]. In adult women the normal vaginal $\mathrm{pH}$ range is 3.8-4.5, but many lubricants and moisturizers exceed these limits. Lubricants with too high or too low $\mathrm{pH}$ may be unacceptable for women. Moreover, additional ingredients can cause mucosal irritation, burning and vaginal discharge. Widely used glycols and microbicides can cause inflammation of vaginal mucosa and alter the vaginal microbiome. Animal studies showed exfoliation of the epithelial cells and mucosal damage caused by nonoxynol-9 [37, 38]. Furthermore, nonoxynol-9 can promote sexual transmission of HIV and other sexually transmitted diseases due to damage of the epithelium. In summary, the WHO recommends the use of lubricants and moisturizers with osmolality under $380 \mathrm{mOsm} / \mathrm{kg}$, but the upper limit of $1200 \mathrm{mOsm} / \mathrm{kg}$ can be acceptable. The $\mathrm{pH}$ of lubricants should lie in the range of vaginal $\mathrm{pH}$. The contents of glycerol should not exceed $9.9 \%$ mass fraction and the use of nonoxynol-9 is not recommended [36].

\section{Laser devices}

Although several trials have revealed positive laser therapeutic effect on GSM symptoms, the use of laser devices remains controversial. The limitations of these studies include short follow-up, small cohorts and lack of a control group. The mechanism of action on vaginal mucosa is different for $\mathrm{CO}_{2}$ and erbium: YAG lasers, although the ultimate impact on the epithelium is similar for both of these devices. Histological examination of the vaginal mucosa after laser treatment revealed changes in all epithelial layers. The thickening of epithelium with cell proliferation in the basal layer as well as increased glycogen storage in the intermediate and superficial cells was noted. Structural changes in the epithelium were accompanied by formation of new blood capillaries penetrating inside newly connective tissue papillae, supporting fibroblasts for enhanced collagen synthesis. These changes in the mucosal structure result in collagen remodeling leading to vaginal rejuvenation [39]. Histological findings have been confirmed by clinical observations. Objective assessment revealed improvement in the VHI (Vaginal Health Index) as well as in the VMI (Vaginal Maturation Index) and MV (Maturity Value) [40]. An increased amount of Lactobacillus in the vaginal microflora resulting in decreased $\mathrm{pH}$ of the vaginal fluid was also reported [41]. Positive subjective evaluation of the therapy was also reported in several trials. Significant improvement in vaginal dryness, itching, burning and dyspareunia compared to baseline was noted [42, 43]. A review of six articles concerning sexual functions in women suffering from GSM treated with $\mathrm{CO}_{2}$ laser revealed statistically significant improvement in FSFI (Female Sexual Function Index) for each single domain and the overall score compared to the entry [44]. Moreover, the out-of-pocket expenses associated with the therapy do not affect patients' satisfaction [45]. The real mechanism of action of the lasers in GSM treatment remains unclear. Probably the beneficial effect is a combination of morphological and biochemical changes in the epithelium assisted by the expression of specific cytokines involved in the anti-inflammatory process. Becorpi et al. observed a significant decrease in the levels of pro-inflammatory cytokines, such as IL-2 and IL-7, as well as inflammatory factors of cell-mediated immunity, in particular RANTES, GM-CSF, and eotaxin, showing the ability of laser therapy to inhibit an inflammatory reaction. On the other hand, they noted an increased concentration of some cytokines and growth factors, such as IL-8, CTACK, LIF, and M-CSF, which are usually involved in inflammatory processes but also stimulate cell proliferation and migration. The authors suggest that increased levels of these molecules could be a consequence of the vaginal tissue repair and remodeling after laser therapy [46]. Recent research has concentrated on novel treatment protocols. Mothes et al. introduced an innovative dual-phase protocol for pulsed ablative erbium: YAG laser treatment of GSM. The aim of the first, ablative phase is to create small canals in the superficial layer of the vaginal epithelium. This phase is followed immediately by the second phase, which induces hyperthermia that irritates collagen fibers in the lamina propria, stimulating neocollagenesis. The authors observed significant improvement in $\mathrm{VHI}$ and vaginal $\mathrm{pH}$ after treatment assisted by high patients' satisfaction [47]. Athanasiou et al. evalu- 
ated the efficacy of additional (extra $4^{\text {th }}$ and extra $5^{\text {th }}$ ) $\mathrm{CO}_{2}$ laser sessions for the management of GSM. They observed significant improvement after the $5^{\text {th }}$ session as compared to the $3^{\text {rd }}$ one in all GSM symptoms. This study could indicate possible dose-dependent effectiveness of GSM treatment [48]. Conversely, the efficacy of the therapy does not seem to be dependent on the power of the device (30 W vs. 40 W) [49]. Recently Gaspar et al. released a pilot study concerning the treatment of urinary symptoms with an intraurethral erbium: YAG laser. The authors observed a reduction of the quantity of leaked urine by $42 \%$ and an improvement by an average of $40 \%$ at 6-month follow-up [50].

\section{Radiofrequency devices}

Radiofrequency (RF) devices generate an electrical field resulting in creation of heat delivered to a tissue. At local temperature of $40-45^{\circ} \mathrm{C}$ the inflammation cascade is initiated and fibroblasts are stimulated to produce collagen, leading to the skin tightening [51]. RF devices have FDA clearance for use in dermatological and surgical procedures (hemostasis and coagulation) but they are not approved for GSM treatment. Observational studies based on the VLQ (Vaginal Laxity Questionnaire), FSFI (Female Sexual Function Index) and FSDS (Female Sexual Distress Scale) revealed improvement in vaginal laxity, arousal, orgasm and sexual satisfaction at 6-month follow-up, but this improvement diminished at 12 months $[52,53]$. The multicenter RCT study VIVEVE showed improvement in vaginal laxity in $43 \%$ of participants in the treated group at 6 months based on VLQ; however, similar improvement was reported in $19.6 \%$ of patients in the sham group. Although the difference between the groups was significant, the placebo effect was greater than expected. Women in the active group had significantly greater improvement in the overall FSFI scale compared to controls. The AE included vaginal discharge, vaginal discomfort and feeling "hot". AEs were reported in both groups, without a significant difference [54]. RF devices work in general in non-ablative mode. However, recently a pilot study evaluating microablative fractional radiofrequency in the treatment of GSM was performed as a proposal of a new therapeutic option. The procedure is performed with a vaginal pen with 64 microneedles inserted into the vagina under direct vision using a vaginal speculum lightly touching the mucosa. A significant improvement was seen in sexual functions and health-related quality of life. All patients stopped using lubricants after treatment. In the satisfaction questionnaire $100 \%$ of participants stated that they were satisfied or very satisfied and $92.6 \%$ felt cured or much better. The limitations of this study were the small number of patients and short follow-up. Further studies are required to establish the long-term effectiveness of this procedure [55].

Long-term, well-designed prospective randomized and comparative trials are needed to assess the therapeutic potential of laser devices in the treatment urogenital dysfunctions. The preliminary observations are promising but currently there is insufficient evidence to promote this therapy in stress urinary incontinence or vaginal laxity [56]. Several other issues should be determined, e.g. ablative versus non-ablative technology, the optimal depth of micro-ablation, and the temperature of the energy. The understanding of the mechanism and physiology of rejuvenation seem to be fundamental in the development of novel treatment protocols. A meta-analysis of 14 studies involving 542 participants showed the improvement of quality of life, reduction of GSM symptoms and positive structural changes in the vaginal mucosa. However, the body of evidence was assessed as "low" or "very low" [57].

In July 2018 the FDA released a safety communication against use of energy-based devices to perform vaginal rejuvenation [58]. This warning was supported by the statement of the International Society for the Study of Vulvovaginal Disease (ISSVD) and the International Continence Society (ICS) highlighting the complexity of postmenopausal sexuality not limited to vaginal mucosa disorders [59]. Further studies are necessary to establish the indications for use of energybased devices in the therapy of vaginal atrophy and for treatment of vulvodynia, lichen sclerosus and urinary incontinence [60].

\section{Conclusions}

GSM constitutes a complex of signs and symptoms that in a very individual manner negatively affects the life of postmenopausal women. Its impact on the quality of life could be modified by cultural, social and psychological factors. Therefore, the therapy must be individually tailored. Among many treatment modalities none is good for all. Every treatment option has its restrictions, contraindications, more or less serious adverse effects and a limited period of improvement or cure. Thus, the problem of GSM so far has remained unsolved. Further research on vaginal biology and physiology as well as on the influence of different therapeutic modes on vaginal tissue is required. At the other end of this spectrum, studies concerning GSM therapy should consider the whole personality of a woman and not concentrate only on the vagina.

\section{Disclosure}

The authors report no conflict of interest. 


\section{References}

1. Moral E, Delgado JL, Carmona F, et al. Genitourinary syndrome of menopause. Prevalence and quality of life in Spanish postmenopausal women. The GENISSE study. Climacteric 2018; 21: 167-173.

2. Palma F, Volpe A, Villa P, et al. Vaginal atrophy of women in postmenopause. Results from a multicentric observational study: The AGATA study. Maturitas 2016; 83: 40-44.

3. Nappi RE, Palacios S, Panay N, et al. Vulvar and vaginal atrophy in four European countries: evidence from the European REVIVE Survey. Climacteric 2016; 19: 188-197.

4. Chua Y, Limpaphayom KK, Cheng B, et al. Genitourinary syndrome of menopause in five Asian countries: results from the Pan-Asian REVIVE survey. Climacteric 2017; 20: 367-373.

5. Nappi RE, de Melo NR, Martino M, et al. Vaginal Health: Insights, Views \& Attitudes (VIVA-LATAM): results from a survey in Latin America. Climacteric 2018; 21: 397-403.

6. Sturdee DW, Panay N. Recommendations for the management of postmenopausal vaginal atrophy. Climacteric 2010; 13: 509-522.

7. Burger HG, Dudley EC, Cui J, et al. A Prospective Longitudinal Study of Serum Testosterone, Dehydroepiandrosterone Sulfate, and Sex Hormone-Binding Globulin Levels through the Menopause Transition. J Clin Endocrinol Metab 2000; 85: 2832-2838.

8. Traish AM, Vignozzi L, Simon JA, et al. Role of Androgens in Female Genitourinary Tissue Structure and Function: Implications in the Genitourinary Syndrome of Menopause. Sex Med Rev 2018; 6: 558-571.

9. Deng Q, Zhang Z, Wu Y, et al. Non-Genomic Action of Androgens is Mediated by Rapid Phosphorylation and Regulation of Androgen Receptor Trafficking. Cell Physiol Biochem 2017; 43: 223-236.

10. Bélanger A, Pelletier G, Labrie F, et al. Inactivation of androgens by UDPglucuronosyltransferase enzymes in humans. Trends Endocrinol Metab 2003; 14: 473-479.

11. Labrie F, Bélanger A, Pelletier G, et al. Science of intracrinology in postmenopausal women. Menopause 2017; 24: 702-712.

12. Labrie F, Cusan L, Gomez JL, et al. Effect of intravaginal DHEA on serum DHEA and eleven of its metabolites in postmenopausal women. J Steroid Biochem Mol Biol 2008; 111: 178-194.

13. Pickar JH, Amadio JM, Bernick BA, Mirkin S. Pharmacokinetic studies of solubilized estradiol given vaginally in a novel softgel capsule. Climacteric 2016; 19: 181-187.

14. Sauer U, Talaulikar V, Davies MC. Efficacy of intravaginal dehydroepiandrosterone (DHEA) for symptomatic women in the peri- or postmenopausal phase. Maturitas 2018; 116; 79-82.

15. Labrie F, Archer DF, Koltun W, et al. Efficacy of intravaginal dehydroepiandrosterone (DHEA) on moderate to severe dyspareunia and vaginal dryness, symptoms of vulvovaginal atrophy, and of the genitourinary syndrome of menopause. Menopause 2016; 23: 243-256.

16. Portman DJ, Labrie F, Archer DF, et al. Lack of effect of intravaginal dehydroepiandrosterone (DHEA, prasterone) on the endometrium in postmenopausal women. Menopause 2015; 22: 1289-1295.

17. Archer DF, Labrie F, Montesino M, Martel C. Comparison of intravagina $6.5 \mathrm{mg}(0.50 \%)$ prasterone, $0.3 \mathrm{mg}$ conjugated estrogens and $10 \mu \mathrm{g}$ estradiol on symptoms of vulvovaginal atrophy. J Steroid Biochem Mol Biol 2017; 174: 1-8.

18. Labrie F, Archer DF, Bouchard C, et al. Prasterone has parallel beneficial effects on the main symptoms of vulvovaginal atrophy: 52-week openlabel study. Maturitas 2015; 81: 46-56.

19. Management of symptomatic vulvovaginal atrophy: 2013 position statement of The North American Menopause. Menopause 2013; 20: 888-902.

20. Archer DF, Kimble TD, Lin FDY, et al. A Randomized, Multicenter, DoubleBlind, Study to Evaluate the Safety and Efficacy of Estradiol Vaginal Cream $0.003 \%$ in Postmenopausal Women with Vaginal Dryness as the Most Bothersome Symptom. J Womens Health 2018; 27: 231-237.

21. Mueck AO, Ruan X, Prasauskas V, et al. Treatment of vaginal atrophy with estriol and lactobacilli combination: a clinical review. Climacteric 2018; 21: 140-147.

22. Santen RJ. Vaginal administration of estradiol: effects of dose, preparation and timing on plasma estradiol levels. Climacteric 2015; 18: 121-134.

23. North American Menopause Society. The role of local vaginal estrogen for treatment of vaginal atrophy in postmenopausal women: 2007 posi- tion statement of The North American Menopause Society. Menopause 2007; 14: 357-369.

24. The NAMS 2017 Hormone Therapy Position Statement Advisory Panel. The 2017 hormone therapy position statement of The North American Menopause Society. Menopause 2017; 24: 728-753.

25. Benoit T, Valera MC, Fontaine C, et al. Estetrol, a Fetal Selective Estrogen Receptor Modulator, Acts on the Vagina of Mice through Nuclear Estrogen Receptor $\alpha$ Activation. Am J Pathol 2017; 187: 2499-2507.

26. Bachmann GA, Komi JO. Ospemifene effectively treats vulvovaginal atrophy in postmenopausal women: results from a pivotal phase 3 study. Menopause 2010; 17: 480-486.

27. Portman DJ, Bachmann GA, Simon JA. Ospemifene, a novel selective estrogen receptor modulator for treating dyspareunia associated with postmenopausal vulvar and vaginal atrophy. Menopause 2013; 20: 623630.

28. Goldstein SR, Bachmann GA, Koninckx PR. Ospemifene 12-month safety and efficacy in postmenopausal women with vulvar and vaginal atrophy. Climacteric 2014; 17: 173-182.

29. Simon JA, Altomare C, Cort S, et al. Overall Safety of Ospemifene in Postmenopausal Women from Placebo-Controlled Phase 2 and 3 Trials. J Womens Health 2018; 27: 14-23.

30. Komi J, Heikkinen J, Rutanen M, et al. Effects of ospemifene, a novel SERM, on biochemical markers of bone turnover in healthy postmenopausal women. Gynecol Endocrinol 2004; 18: 152-158.

31. Constantine GD, Goldstein SR, Archer DF. Endometrial safety of ospemifene: results of the phase $2 / 3$ clinical development program. Menopause 2015; 22: 36-43.

32. Lumsden MA. The NICE Guideline - Menopause: diagnosis and management. Climacteric 2016; 19: 426-429.

33. Hirsch HD, Shih E, Thacker HL. ERAAs for menopause treatment: Welcome the "designer estrogens". Cleve Clin J Med 2017; 84: 463-470.

34. Bachmann G, Bobula J, Mirkin S. Effects of bazedoxifene/conjugated estrogens on quality of life in postmenopausal women with symptoms of vulvar/vaginal atrophy. Climacteric 2010; 13: 132-140.

35. Edwards D, Panay N. Treating vulvovaginal atrophy/genitourinary syndrome of menopause: how important is vaginal lubricant and moisturizer composition? Climacteric 2016; 19: 151-161.

36. Use and procurement of additional lubricants for male and female condoms: WHO/UNFPA/FHI360 Advisory note. World Health Organization 2012.

37. Fashemi B, Delaney ML, Onderdonk AB, Fichorova RN. Effects of feminine hygiene products on the vaginal mucosal biome. Microb Ecol Health Dis 2013; 24.

38. Dayal MB, Wheeler J, Williams CJ, Barnhart KT. Disruption of the upper female reproductive tract epithelium by nonoxynol-9. Contraception 2003; 68: 273-279.

39. Zerbinati N, Serati M, Origoni M, et al. Microscopic and ultrastructural modifications of postmenopausal atrophic vaginal mucosa after fractional carbon dioxide laser treatment. Lasers Med Sci 2015; 30: 429-436.

40. Gambacciani $M$, Levancini $M$, Cervigni $M$. Vaginal erbium laser: the second-generation thermotherapy for the genitourinary syndrome of menopause. Climacteric 2015; 18: 757.

41. Athanasiou S, Pitsouni E, Antonopoulou S. The effect of microablative fractional $\mathrm{CO} 2$ laser on vaginal flora of postmenopausal women. Climacteric 2016; 19: 512-518.

42. Arroyo C. Fractional CO2 laser treatment for vulvovaginal atrophy symptoms and vaginal rejuvenation in perimenopausal women. Int J Womens Health 2017; 9: 591-595.

43. Gambacciani M, Levancini M, Russo E, et al. Long-term effects of vaginal erbium laser in the treatment of genitourinary syndrome of menopause. Climacteric 2018; 21: 148-152.

44. Salvatore S, Pitsouni E, Del Deo F, et al. Sexual Function in Women Suffering From Genitourinary Syndrome of Menopause Treated With Fractionated CO2 Laser. Sex Med Rev 2017; 5: 486-494.

45. Lang P, Dell JR, Rosen L, et al. Fractional CO2 laser of the vagina for genitourinary syndrome of menopause: Is the out-of-pocket cost worth the outcome of treatment? Lasers Surg Med 2017; 49: 882-885.

46. Becorpi A, Campisciano G, Zanotta N. Fractional CO2 laser for genitourinary syndrome of menopause in breast cancer survivors: clinical, immunological, and microbiological aspects. Lasers Med Sci 2018; 33: 1047-1054. 
47. Mothes AR, Runnebaum M, Runnebaum IB. An innovative dual-phase protocol for pulsed ablative vaginal Erbium:YAG laser treatment of urogynecological symptoms. Eur J Obstet Gynecol Reprod Biol 2018; 229: 167-171.

48. Athanasiou S, Pitsouni E, Falagas ME, et al. CO 2 -laser for the genitourinary syndrome of menopause. How many laser sessions? Maturitas 2017; 104: 24-28.

49. Pitsouni E, Grigoriadis T, Falagas M, et al. Microablative fractional CO2 laser for the genitourinary syndrome of menopause: power of 30 or $40 \mathrm{~W}$ ? Lasers Med Sci 2017; 32: 1865-1872.

50. Gaspar A, Maestri S, Silva J, et al. Intraurethral Erbium: YAG laser for the management of urinary symptoms of genitourinary syndrome of menopause: A pilot study. Lasers Surg Med 2018; 50: 802-807.

51. Qureshi AA, Tenenbaum MM, Myckatyn TM. Nonsurgical Vulvovaginal Rejuvenation With Radiofrequency and Laser Devices: A Literature Review and Comprehensive Update for Aesthetic Surgeons. Aesthet Surg J 2018; 38: 302-311.

52. Sekiguchi Y, Utsugisawa Y, Azekosi Y, et al. Laxity of the Vaginal Introitus After Childbirth: Nonsurgical Outpatient Procedure for Vaginal Tissue Restoration and Improved Sexual Satisfaction Using Low-Energy Radiofrequency Thermal Therapy. J Womens Health 2013; 22: 775-781.

53. Millheiser LS, Pauls RN, Herbst SJ, Chen BH. Radiofrequency Treatment of Vaginal Laxity after Vaginal Delivery: Nonsurgical Vaginal Tightening. J Sex Med 2010; 7: 3088-3095.

54. Krychman M, Rowan CG, Allan BB, et al. Effect of Single-Treatment, Surface-Cooled Radiofrequency Therapy on Vaginal Laxity and Female Sexual Function: The VIVEVE I Randomized Controlled Trial. J Sex Med 2017; 14: 215-225.

55. Kamilos MF, Borrelli CL. New therapeutic option in genitourinary syndrome of menopause: pilot study using microablative fractional radiofrequency. Einstein São Paulo 2017; 15: 445-451.

56. Lang P, Karram M. Lasers for pelvic floor dysfunctions: is there evidence? Curr Opin Obstet Gynecol 2017; 9: 354-358.

57. Pitsouni E, Grigoriadis T, Falagas ME, et al. Laser therapy for the genitourinary syndrome of menopause. A systematic review and meta-analysis. Maturitas 2017; 103: 78-88.

58. FDA Warns Against Use of Energy-Based Devices to Perform Vaginal 'Rejuvenation' or Vaginal Cosmetic Procedures: FDA Safety Communication. https://www.fda.gov/medicaldevices/safety/alertsandnotices/ ucm615013.htm (access: 28.10.2018)

59. ISSVDICS comments on the FDA communication on the use of energybased devices to perform vaginal "rejuvenation" or vaginal cosmetic procedures. https://3b64we1rtwev2ibv6q12s4dd-wpengine.netdna-ssl. com/wp-content/uploads/2018/08/2018_08_05-ISSVD_ICS.pdf (access: 28.10.2018).

60. Tadir Y, Gaspar A, Lev-Sagie A, et al. Light and energy based therapeutics for genitourinary syndrome of menopause: Consensus and controversies. Lasers Surg Med 2017; 49: 137-159. 